Proc. XII Int. School on Theoretical Physics — Symmetry and Structural Properties of Condensed Matter

\title{
New Type of Composite Gyrotropic Metamaterial ${ }^{\dagger}$
}

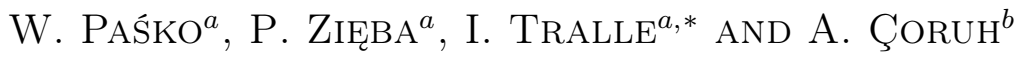 \\ ${ }^{a}$ Faculty of Mathematics and Natural Sciences, Theoretical Physics Department, University of Rzeszów, \\ S. Pigonia 1 A0/B1, 35-310 Rzeszów, Poland \\ ${ }^{b}$ Faculty of Arts and Science, Department of Physics, Sakarya University, Sakarya, Turkey

\begin{abstract}
In this work we examined the possibility of fabricating the metamaterial in a relatively simple way. Our idea was to use the three-component mixture of ingredients, where one of them is responsible for the negative permeability $\mu(\omega)$ of hypothetical metamaterial, while the other two cause the negative value of effective permittivity $\varepsilon(\omega)$. In our previous work, we considered $\mathrm{Hg}_{1-x} \mathrm{Cd}_{x} \mathrm{Te}$ semiconductor compound as one of the ingredients of mixture. As fabrication of the $\mathrm{Hg}_{1-x} \mathrm{Cd}_{x}$ Te is related to using mercury which is very poisoning, we tried to exclude this material. In the work we proved by numerical simulations the possibility of substituting mercury cadmium telluride by $\mathrm{Pb}_{1-x} \mathrm{Sn}_{x}$ Te. We have shown by computer simulations that by the proper fitting of the parameters, e.g. the radius of nanoparticles, their magnetic moments, the relative concentration of ingredients, etc., it is possible to obtain the metamaterial with negative refraction index in a relatively broad range of temperatures and magnetic fields. The last seems to be very promising in terms of practical applications of metamaterials.
\end{abstract}

DOI: 10.12693/APhysPolA.132.121

PACS/topics: 81.05.Xj, 81.05.Zx, 78.67.Pt, 42.70.-a

\section{The model}

The model used in the paper is basically the same as in our previous work [1], where the theoretical and numerical examinations of three-component metamaterial have been done. As we have shown there, the mixture of appropriately chosen three components, where one of them is responsible for the magnetic properties of mixture, and the other two contribute to the negativity of real part of effective permittivity (in some range of frequency domain), may result in a negative index of refraction of such medium. We assumed also that the magnetic nanoparticles responsible for the negativity of real part of magnetic permeability, are the grains of the spherical form.

As it was mentioned in Sect. 1, in paper [1] for one of the mixture components there was chosen $\mathrm{Hg}_{1-x} \mathrm{Cd}_{x} \mathrm{Te}$ semiconductor compound. The point however is that in the fabrication process of $\mathrm{Hg}_{1-x} \mathrm{Cd}_{x}$ Te there involves mercury, which is very poisoning. That is why it is desirable to find the material whose electrical properties are similar to those of $\mathrm{Hg}_{1-x} \mathrm{Cd}_{x} \mathrm{Te}$, but which does not contain Hg. Then, one should carry out the corresponding computer simulations in order to establish the domains of existence of the hypothetical metamaterial, which could be produced on such a basis. It turns out that the materials similar to $\mathrm{Hg}_{1-x} \mathrm{Cd}_{x} \mathrm{Te}$, regarding its electrical properties do really exist; it can be for instance, $\mathrm{Pb}_{1-x} \mathrm{Sn}_{x} \mathrm{Te}$.

\footnotetext{
*corresponding author; e-mail: tralle@ur.edu.pl

${ }^{\dagger}$ This work is the continuation of our study started in: I. Tralle, P. Zięba, W. Paśko, J. Appl. Phys. 115, 233509 (2014)
}

\section{Numerical results}

The expressions for permittivities for silver particles $\left(\varepsilon_{1}(\omega)\right)$ and magnetic nanoparticles $\left(\varepsilon_{2}(\omega)\right)$ were derived in [1]. One should note here that due to the fact that Ag is a very good conductor, we have used the Drude theory, whereas to obtain the relation for $\left(\varepsilon_{3}(\omega)\right)$ we assumed the intermediate value of its real part and added the imaginary part of it in the following way: $\varepsilon_{3}(\omega)=12.5+4 \mathrm{i}$ (see e.g. $[2,3]$ ).

The third component of our mixture, i.e. $\mathrm{Pb}_{1-x} \mathrm{Sn}_{x} \mathrm{Te}$, is characterized by different conductivity properties (which vary from the semiconductor to semimetal phase) depending on tin's percentage content. As it was stated above, from the point of view of electron transport properties, $\mathrm{Pb}_{1-x} \mathrm{Sn}_{x} \mathrm{Te}$ is similar to $\mathrm{Hg}_{1-x} \mathrm{Cd}_{x} \mathrm{Te}$. That is, the concentration of free charge carriers (electrons) in it depends strongly on the tin's concentration. So, in order to calculate the $\mathrm{Pb}_{1-x} \mathrm{Sn}_{x}$ Te-permittivity we should take into account the contribution which stems from the presence of free charge carriers. After using the Drude model (we can do this, because we can express permittivity in terms of conductivity) we obtain

$$
\varepsilon_{2}(\omega)=\varepsilon_{\infty}(x)-\frac{1}{\varepsilon_{0}} \frac{\sigma_{0} \Gamma_{2}}{\omega^{2}+\Gamma_{2}^{2}}+\frac{\mathrm{i} \sigma_{0} \Gamma_{2}^{2}}{\omega\left(\omega^{2}+\Gamma_{2}^{2}\right)} .
$$

The concentration of electrons is calculated, using the following relationship [4]:

$$
\begin{aligned}
& n_{e}(x, T)=\left(8.92-34.46 x+\left(2.25 \times 10^{-3}\right) T\right. \\
& \left.\quad+\left(4.12 \times 10^{-2}\right) x T+97 x^{2} \times 10^{14}\right) E_{g}^{0.75} T^{1.5} \\
& \quad \times \exp \left(-E_{g} /\left(2 k_{B} T\right)\right),
\end{aligned}
$$

where $E_{g}$ (in meV) is the energy gap

$$
E_{g}(x, T)=171.5-535 x+\sqrt{(12.8)^{2}+0.19(T+20)^{2}} .
$$

To get the mobility of electrons in $\mathrm{Pb}_{1-x} \mathrm{Sn}_{x}$ Te, we used the experimental data obtained in [5]. In this work the 
authors present the carriers mobility versus temperature for different values of tin content. For our purposes we interpolated the experimental data for boundary values of tin content in $\mathrm{Pb}_{1-x} \mathrm{Sn}_{x}$ Te considered there. The corresponding relations for $x=0.014$ and $x=0.124$ are presented below:

$$
\begin{aligned}
& \mu_{0.014}(T)=345.729+\frac{1.2659 \times 10^{8}}{T^{2}}, \\
& \mu_{0.124}(T)=-118.819+\frac{226105}{T} .
\end{aligned}
$$

The effective mobility of electrons (in $\mathrm{cm}^{2} /(\mathrm{V} \mathrm{s})$ ) for given temperature was obtained by assuming the linear dependence of electron's mobility on Sn-content (in the range between the boundary values) as follows:

$$
\frac{\mu_{\alpha}(T)-\mu(x, T)}{\alpha-x}=\frac{\mu_{\alpha}(T)-\mu_{\beta}(T)}{\alpha-\beta},
$$

where $\alpha=0.014$ and $\beta=0.124$. As a result we have

$$
\begin{aligned}
& \mu(x, T)=\mu_{0.014}(T)-\left(\mu_{0.014}(T)-\mu_{0.124}(T)\right) \\
& \quad \times \frac{x-0.014}{0.124-0.014} .
\end{aligned}
$$

Our primary goal is to find out if metamaterial exists in a broad range of temperatures, but of course, the characteristics of composite is dependent not only on the temperature. The difficulty of the simulation lies in the multidimensionality of the model, because the values of permittivity and permeability are depending on eight parameters: temperature, external magnetic field, radius of nanoparticles, their magnetic moments, tin content in $\mathrm{Pb}_{1-x} \mathrm{Sn}_{x}$ Te and relative concentrations $f_{1}, f_{2}, f_{3}$ of the components in a mixture. Additionally, we must determine the appropriate frequency range for which the composite has the property of being metamaterial. Our objective is to find those combinations of the model parameters, for which the real parts of the permittivity and permeability of the composite are simultaneously negative. From this point of view, it is extremely important to choose an optimal and efficient search strategy.

Our approach is the following. We start with the localization of the frequency range for which the real part of the permeability for our composite is negative. We want to search for the smallest possible value of $\operatorname{Re}(\mu)$. To attain this objective, we calculate the first derivative of $\operatorname{Re}\left(\chi_{+}\right)$, equalize it to zero, and solve the obtained equation for $\omega$. From two roots, we select the greater one, $\omega_{R}$, for which the real part of $\chi_{+}$reaches its minimum. Furthermore, from the properties of $\operatorname{Im}\left(\chi_{+}\left(\omega_{R}\right)\right)$, it follows that $\operatorname{Im}\left(\chi_{+}\left(\omega_{R}\right)\right)>0$. At that point, we make sure that for the frequency $\omega_{R}$ the real part of $\mu$ of the composite is to be negative, otherwise the composite could not be metamaterial. The calculated value of $\omega_{R}$ is determined by properties of third component of composite, i.e. ferromagnetic nanoparticles, whose behaviour in magnetic field is controlled by four parameters of the model, namely the strength of the magnetic field itself, radii of particles, their magnetic moment, and the tem- perature. Having set those four model parameters, we try to choose the remaining ones to get the negative real part of $\epsilon$ of the composite at the same time requiring the imaginary part of $\epsilon$ to be nonnegative. To achieve this goal, we can still use three degree of freedom i.e. we can change $x$, the concentration of tin in $\mathrm{Pb}_{1-x} \mathrm{Sn}_{x} \mathrm{Te}$, and $f_{1}, f_{2}, f_{3}$, relative fractions of components of the composite. Computationally, this is the most demanding part of the entire calculation as it requires testing further combinations of parameter values and evaluating relevant complex formulae (1)-(3). Such searching procedure is much more effective, and faster leads to the goal than to blindly test all possible combinations of values from all parameters set.

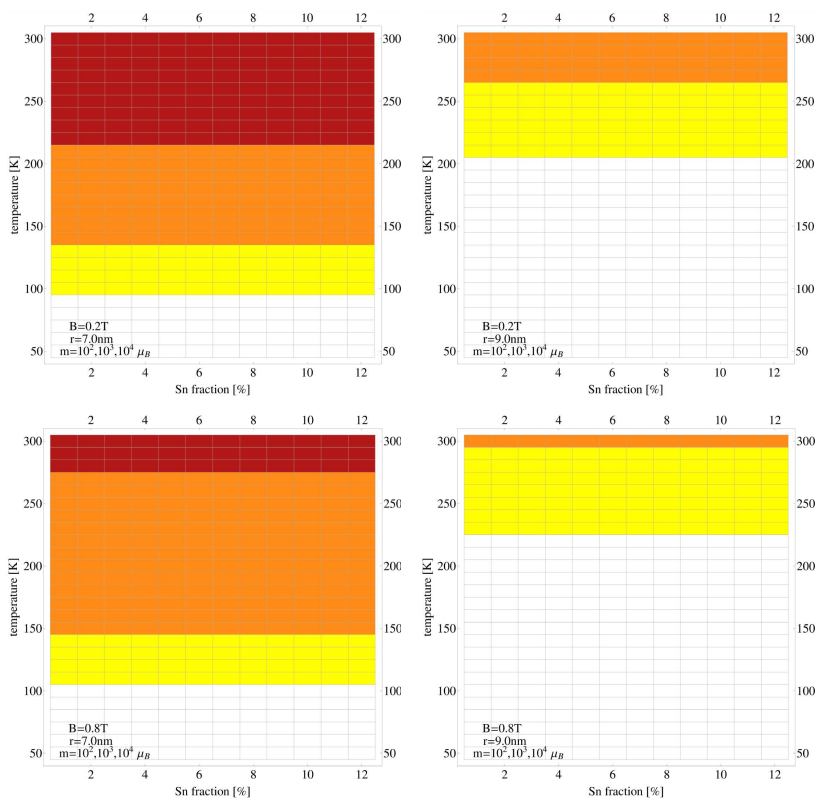

Fig. 1. The regions colored in red, orange, and (see text) in $T-x$ plane and for the different values of model parameters. $B, r$ and $m$ correspond to the domains in which at least one set of the filling factors $f_{1}, f_{2}, f_{3}$ exists, such that the resulting mixture possesses $\operatorname{Re} n<0$.

Figures 1-2 illustrate the results of our simulations. Figure 1 presents in $T-x$ plane, for different parameter values of $B, r$, and ferromagnetic nanoparticles magnetic moment $m$, the domains where at least one set of the filling factors $f_{1}, f_{2}$, and $f_{3}$ exists, such that the resulting mixture has the real part of the refractive index less than zero. The different colours have the following meaning: denoted by $D_{1}$ is the region coloured in red, the domain coloured in orange as $D_{2}$, and the region coloured in yellow as $D_{3}$. Then, $D_{1}$ corresponds to $m=10^{2} \mu_{B}$, $D_{1} \cup D_{2}$ corresponds to $m=10^{3} \mu_{B}$ and $D_{1} \cup D_{2} \cup D_{3}$ corresponds to $m=10^{4} \mu_{B}$. We observe that the greater value of nanoparticle magnetic moment is, the greater is the domain of metamaterial existence. In Fig. 2 we present the real and imaginary parts of refraction index of the hypothetical metamaterial as some kind of "phase diagrams", where the values of them are shown in the 

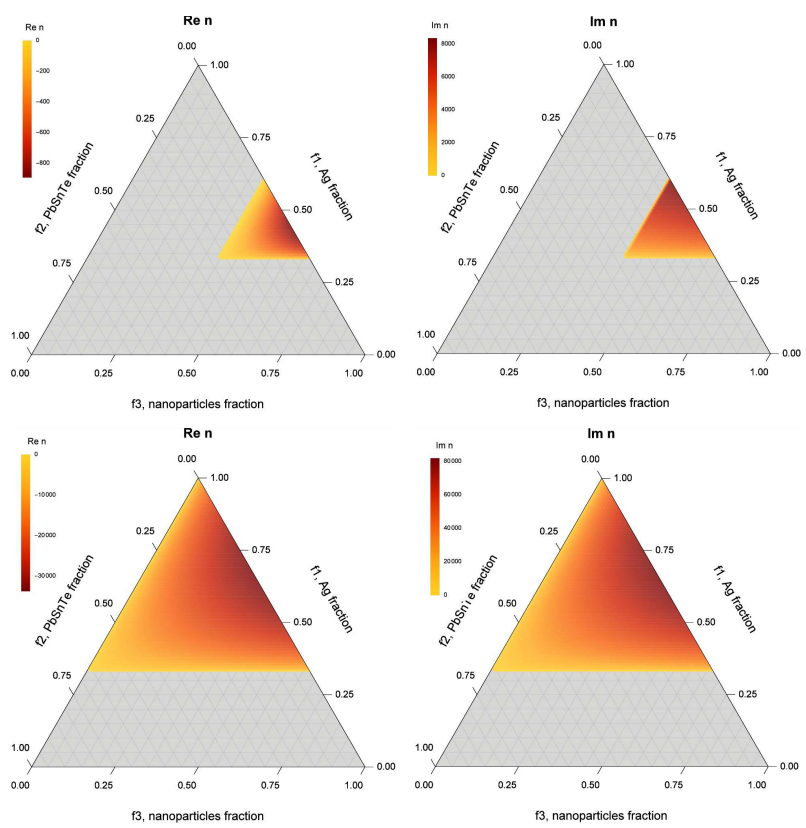

Fig. 2. Real and imaginary parts of the refractive index of the composite for selected cases presented on the ternary diagram. On each axis of the triangle the relative contributions of individual components of the composite are indicated, which are expressed as the values of the filling factors $f_{1}, f_{2}, f_{3}$. Model parameters: $B=0.2 \mathrm{~T}, r=8 \mathrm{~nm}, m=10^{4} \mu_{\mathrm{B}}, T=300 \mathrm{~K}, x=0.08$ (the first row); $B=0.4 \mathrm{~T}, r=8 \mathrm{~nm}, m=10^{4} \mu_{\mathrm{B}}$, $T=300 \mathrm{~K}, x=0.08$ (the second row).

form of a triangular colour maps and where the edges of triangles correspond to the changing of the $f_{1}, f_{2}, f_{3}$, the relative fractions of components of the composite.

Of course as it usually happens, if produced, such a metamaterial will show its disadvantages or limitations in use, too. First of all, a negative refraction can be achieved only if the material is in an external, although moderate magnetic field. In our calculations, $B$ was restricted to $0.8 \mathrm{~T}$. On the other hand, in some circumstances it could be an advantage, since switching magnetic field on and off, one can trigger on/off the left-handedness or rather, the state of matter when the material becomes metamaterial. The metamaterial which was examined by us previously [1] and which contained $\mathrm{Hg}_{1-x} \mathrm{Cd}_{x} \mathrm{Te}$, in most of the cases studied by us exhibited relatively great imaginary parts of $\varepsilon$ and $\mu$, which means that it would be characterized by significant losses. Sometimes one can encounter with the statement that the losses render photonic negative refraction-index media useless [6]. Recently however, some authors argued $[7,8]$ that the losses in metamaterials can be used for developing new and interesting devices, too.

\section{Conclusions}

We have shown that it is possible to substitute such material as $\mathrm{Hg}_{1-x} \mathrm{Cd}_{x} \mathrm{Te}$, whose fabrication is dangerous in regard of mercury, by $\mathrm{Pb}_{1-x} \mathrm{Sn}_{x}$ Te which is much less poisoning for the environment. In the work we carried out computer simulations in the frame of the proposed model in order to establish the domains of existence of such material, searching through a vast parameter space. We have seven parameters to be controlled in course of simulations, these are the temperature, external magnetic field, radius of nanoparticles, their magnetic moments, fraction of tin in $\mathrm{Pb}_{1-x} \mathrm{Sn}_{x}$ Te-compound, and the relative concentrations $f_{1}, f_{2}$ and $f_{3}$ of the components in a mixture. As a result, the domains where the material becomes negative refraction index material, were established relatively to all parameters characterizing the mixture.

\section{Acknowledgments}

This work has been done due to the support which we have got from the Centre for Innovation and Transfer of Natural Science and Engineering Knowledge. P.Z. would like to acknowledge the Interdisciplinary Centre for Computational Modelling in the University of Rzeszów for the possibility of performing computations (the computational grant: G-030).

\section{References}

[1] I. Tralle, P. Zięba, W. Paśko, J. Appl. Phys. 115 , 233509 (2014).

[2] T. Mackay, A. Lakhtakia, Opt. Commun. 234, 35 (2004).

[3] A.J. Duncun, T. Mackay, A. Lakhtakia, Opt. Commun. 271, 470 (2007).

[4] A. Rogalski, M. Jóźwikowski, Phys. Status Solidi 111, 559 (1989)

[5] J. Konig, J. Nurnus, R. Glatthaar, H. Bottner, A Lambrecht, in: Proc. 8th European Workshop on Thermoelectrics, Kraków (Poland), 2004.

[6] N.I. Zheludev, Science 328, 582 (2010).

[7] N.I. Landy, S. Sajuyigbe, J.J. Mock, D.R. Smith, W.J. Padilla, Phys. Rev. Lett. 100, 207402 (2008).

[8] F. Ding, Y. Cui, X. Ge, Y. Jin, S. He, Appl. Phys. Lett. 100, 103506 (2012). 